\title{
Nutrition in pregnancy: testing the effects of the diet of the poorest socioeconomic group in Scotland on the liver of pregnant rats
}

\author{
S.M. Hay, H. Dasgin and W.D. Rees \\ Rowett Institute, University of Aberdeen, Foresterhill Aberdeen, AB25 2ZD, Scotland UK
}

The liver plays an important role in modulating lipid metabolism. Surplus dietary carbohydrates can be converted into fat by de novo lipogenesis (DNL). Alternatively, excess lipid can be oxidised for energy. Pregnant women in deprived populations in Scotland consume diets high in saturated fat and refined carbohydrates ${ }^{(1)}$. We have used an animal model to examine how the maternal liver adapts to this imbalance in pregnancy by measuring the expression of genes regulating the synthesis and oxidation of fatty acids.

An experimental diet, formulated to reflect the median intakes of pregnant women in tenth decile of the Scottish index of multiple deprivation (SIMD) ${ }^{(1)}$ was compared with a second experimental diet, formulated to meet the recommendations of the Scientific Advisory Committee on Nutrition (SACN), i.e. low in saturated fat and sugar and with micronutrients adjusted to meet the recommended intakes. Three groups of Hooded-Lister strain female rats were fed either the SIMD, SACN or AIN-93 G (reference) diets for an adaptation period of 3 weeks and then after being mated with normal males, continued to be fed the experimental diets until they were killed on day 21 of gestation. Samples of the maternal liver were recovered, and the relative abundance of mRNA species $(n=6$ per group) was measured by quantitative RT-PCR as described previously ${ }^{(2)}$. Data was analysed by one-way ANOVA followed by Fisher's multiple comparison test.

The abundance of the mRNAs coding for acetyl CoA carboxylase (Acc-1) and fatty acid synthase (FAS) was unchanged in the liver of animals fed the SIMD diet compared with animals fed the SACN or AIN diets. These enzymes are key stages in DNL from carbohydrate, suggesting that the additional free sugar in the diet did not increase the synthesis of fatty acids. There was a two-fold increase $(\mathrm{P}=0.009)$ in the mRNA for liver-type carnitine palmitoyl transferase (L-CPT) only in the SIMD group. This protein transfers fatty acids to the mitochondria, suggesting that there is an increase in beta-oxidation in response to additional saturated fat in the diet.

We also examined the mRNAs coding for transcriptional regulators. There was a 1.8 fold increase in the mRNAs for the alpha and gamma isoforms of the peroxisome proliferator-activated receptor (PPAR- $\alpha, \mathrm{P}=0.011$ and PPAR- $\gamma \mathrm{P}=0.002)$ in the SIMD group when compared with SACN and AIN. In contrast the relative abundance of the mRNA for the sterol response element binding protein (SREBP-1c) was similar in all three groups.

This pattern is unique and different from other diet models such as methyl deficiency ${ }^{(2)}$ or low protein and indicates complex interactions between nutrition and gene expression in the maternal liver. Changes in hepatic metabolism have an important role in protecting the developing fetus from imbalances in the maternal diet.

1. Haggarty P, Campbell DM, Duthie S, et al. (2009) Br J Nutr 102, 1487-97.

2. McNeil CJ, Hay SM, Rucklidge G, et al. (2008) Br J Nutr 99, 262-71. 